\title{
Fertility preservation procedures in girls, adolescents and women
}

\author{
Bettina Böttcher (D) · Maren Goeckenjan
}

Received: 16 April 2020 / Accepted: 5 May 2020 / Published online: 20 May 2020

(C) The Author(s) 2020

\begin{abstract}
Summary Fertility preservation has become an integral part of standard treatment for young patients with planned gonadotoxic therapy. The decision-making process is an interdisciplinary challenge to both oncologists and radiotherapists involved in the process of diagnosis and treatment. Nevertheless, even today, not every patient receives adequate counselling about fertility preserving techniques. Current data on the options of fertility preservation for women, including cryopreservation of oocytes, embryos, ovarian tissue, transposition of ovaries and medical treatment, are summarized in this review.
\end{abstract}

Keywords Premature ovarian insufficiency · Ovarian tissue cryopreservation - Autotransplantation · GnRH analogues · Counselling

\section{Introduction}

Fertility preservation has become an emerging field in recent decades and is of enormous importance for patients of reproductive age. Due to improved therapies and increased life expectancies, parenthood is increasingly becoming the focus of attention among young cancer survivors. Fertility preservation has proven to be an interdisciplinary challenge that involves oncologists, radiotherapists, pediatricians, surgeons, urologists, psychologists, reproductive endocrinologists and others. Before starting gonadotoxic

\section{Dr. B. Böttcher, MA (凶)}

Department of Gynecological Endocrinology and

Reproductive Medicine, Medical University of Innsbruck, Anichstraße 35, 6020 Innsbruck, Austria

bettina.boettcher@i-med.ac.at

Dr. M. Goeckenjan

Department of Gynecology and Obstetrics, University Hospital Carl Gustav Carus, Technische Universitaet Dresden, Dresden, Germany therapies, patients need to be informed about their individual options for fertility preservation according to the underlying disease, planned treatment, individual ovarian reserve, prognosis and personal desires.

Chemotherapy, radiotherapy and surgery can lead to premature ovarian insufficiency (POI) in girls and women. Several factors influence the severity of ovarian damage. These include the age of the girl or woman at the time of treatment, the individual ovarian reserve prior to treatment, the agents and dosage of the chemotherapy as well as the location of exposure and dose of radiation.

Established procedures include embryo and oocyte cryopreservation, cryopreservation of ovarian tissue and transplantation, the application of analogues of gonadotropin releasing hormones (GnRH) and the transposition of the ovaries in the case of radiation. In German-speaking countries, the network "FertiPROTEKT", founded in 2006, has successfully managed to increase awareness among doctors and patients that, in spite of gonadotoxic treatment, fertility preservation methods can increase the chances for a child in the future. A guideline of the Austrian, German and Swiss Societies of Obstetrics and Gynecology on fertility preservation in malignant diseases was recently published [1].

Fertility preservation concerns not only oncologic patients, but all patients whose future fertility might be impaired by genetic disorders or benign diseases requiring therapies that reduce ovarian reserve, like autoimmune diseases or also gynecological diseases such as endometriosis.

The aim of this short review is to provide an update on established techniques of fertility preservation in girls and women. Future perspectives are the subject of the following short review. 


\section{Controlled ovarian stimulation and cryopreservation of oocytes and/or embryos}

Oocyte and embryo cryopreservation have become standard procedures in female cancer patients. Established techniques of in vitro fertilization (IVF)/ intracytoplasmic sperm injection (ICSI) can be offered to women with a male partner. Cryopreservation of oocytes can be offered to patients without a male partner or not willing to depend on the partner's consent to transfer embryos in the future. The progress of vitrification techniques has improved the outcome of oocyte cryopreservation in recent years [2]. As a matter of course, women can choose to split oocytes to cryopreserve both embryos and unfertilized oocytes.

In both cases, hormonal ovarian stimulation lasting on average 10-14 days is required. Adapted stimulation protocols make it possible to start the stimulation independently of the menstrual cycle and show similar outcomes in terms of the number of retrieved oocytes [3, 4].

Hormonal ovarian stimulation is performed with standard protocols, followed by triggering ovulation and oocyte retrieval, which is performed transvaginally and ultrasound-guided.

In the case of estrogen-dependent malignancies like breast cancer, stimulation protocols with an aromatase inhibitor, letrozole, are recommended to avoid high levels of estrogen [5]. Overall survival and relapse-free survival in patients with breast cancer after stimulation with letrozole and gonadotropins compared to women with breast cancer without fertility preservation procedures did not differ significantly [5].

According to data of the Fertiprotekt network, on average between five and nine oocytes are retrieved [6].

For controlled ovarian stimulation and cryopreservation of oocytes and/or embryos, a delay of oncological treatment by at least 10 days must be considered.

In vitro maturation of oocytes can be an option in patients with a high antral follicle count and insufficient time or contraindications for ovarian stimulation. Immature oocytes are retrieved and maturated in vitro. Unfortunately, the success rate of this technique is limited [7].

\section{Cryopreservation of ovarian tissue and transplantation}

Cryopreservation of ovarian tissue is the option for girls and women whose chemotherapy needs to start immediately and cannot be delayed. Over 130 live births after transplantation of ovarian tissue have been reported worldwide [8]. This technique has long been considered experimental, but, according to the aforementioned guideline of German-speaking countries, cryopreservation of ovarian tissue has become an established option of fertility preservation [1].
The procedure comprises the surgical removal of the ovarian tissue, cryopreservation with slow freezing techniques and-after a certain relapse-free period-transplantation of the ovarian graft. The intervention can be planned cycle-independent, is performed via laparoscopy and chemotherapy can even start on the same day or the day after. It does not require hormonal pretreatment. Furthermore, autotransplantation of ovarian tissue is able to not only restore fertility, but also rescue ovarian endocrine function for later life.

One major risk of cryopreservation of ovarian tissue remains the potential transplantation of malignant cells depending on the type of cancer. This risk is especially high in patients with leukemia, neuroblastoma and Burkitt's lymphoma [9]. Certain criteria need to be met when offering this option: The patient should have a relatively high ovarian reserve and therefore be under the age of 35 years. The prognosis of 5-year survival should be good and the risk of POI should be at least 50\% [8]. At the time of autotransplantation, the fragmented ovarian graft is transplanted either orthotopically in the pelvic cavity onto the ovary or using a peritoneal window or heterotopically outside the pelvic cavity. Ovarian tissue autotransplantation has the advantage of natural conception if fallopian tubes are permeable and ovarian function is restored after transplantation. Observations have shown that ovarian activity starts on average 4 months after transplantation and remains for approximately 5 years. The procedure can usually be repeated, since only small portions of the ovarian tissue are transplanted [10].

In vitro maturation of immature oocytes can be combined with ovarian tissue cryopreservation if time or type of cancer do not allow controlled ovarian stimulation [11]. The oocytes can either be retrieved by puncture of visible antral follicles or from the dissection medium when processing the ovarian tissue [12]. These techniques are still experimental.

\section{Transposition of the ovaries}

Radiotherapy techniques have improved in recent decades, and it is now possible to precisely localize the radiotoxic ovarian effects [13]. Nevertheless, in female patients with planned pelvic radiotherapy, ovarian function can be preserved by transposition out of the radiation field [14]. The laparoscopic transposition of ovaries reduces radiotherapy-induced ovarian damage and may also be performed in prepubertal girls [15]. Risks include operative complications and postoperative ovarian cysts [15]. During decisionmaking, the radiotherapy-related uterine damage that could impair a normal course of pregnancy, with higher risks of missed abortions and preterm births, should also be considered. 


\section{Fertiprotective agents (GnRH analogues)}

The administration of gonadotropin-releasing hormone agonists (GnRHa) to minimize gonadal damage remains a matter of controversy $[1,16]$. Several trials in different cohorts have been performed with conflicting results. Outcome measurements were not standardized and included different parameters, such as the level of anti-Müllerian hormone and/or follicle stimulating hormone (FSH), amenorrhea and pregnancy rates [17].

A prospective randomized controlled study in lymphoma survivors did not report any benefit for GnRHa [18], whereas two prospective studies in women with hormone-receptor negative breast cancer showed encouraging results in terms of higher pregnancy rates [19] and restored ovarian function [19, 20]. A recent Cochrane review stated that $\mathrm{GnRH}$ analogues appeared to be effective with regard to the resumption of menstruation, ovulation and premature ovarian insufficiency, but evidence was limited on pregnancy rates and on the protection of the ovaries [21].

Therefore, GnRH analogues should be considered as an additional method, but not as the only option to preserve fertility [1].

\section{Conclusion}

Female fertility preservation remains a fundamental issue in women with gonadotoxic therapies or women that suffer from diseases or genetic predispositions compromising future fertility. Techniques include oocyte and embryo cryopreservation, ovarian tissue cryopreservation, transposition of the ovary and the administration of GnRHa. These procedures can be combined starting with the cryopreservation of ovarian tissue, followed by hormonal ovarian stimulation and by GnRHa during the course of chemotherapy. Counselling comprises an interdisciplinary approach by oncologists, specialists in reproductive medicine and other disciplines [16, 22] and is a major challenge [23]; nevertheless, women facing premature ovarian failure necessarily need to be informed about options of fertility preservation. Patients and their partners should be enabled to make an autonomous decision on their future reproductive life [24].

\section{Take home message}

- Cancer patients need to be informed about options of fertility preservation.

- The demand for fertility preservation procedures in patients with benign diseases or genetic predispositions is increasing.

Funding Open access funding provided by University of Innsbruck and Medical University of Innsbruck.
Conflict of interest B. Böttcher and M. Goeckenjan declare that they have no competing interests.

Open Access This article is licensed under a Creative Commons Attribution 4.0 International License, which permits use, sharing, adaptation, distribution and reproduction in any medium or format, as long as you give appropriate credit to the original author(s) and the source, provide a link to the Creative Commons licence, and indicate if changes were made. The images or other third party material in this article are included in the article's Creative Commons licence, unless indicated otherwise in a credit line to the material. If material is not included in the article's Creative Commons licence and your intended use is not permitted by statutory regulation or exceeds the permitted use, you will need to obtain permission directly from the copyright holder. To view a copy of this licence, visit http://creativecommons.org/licenses/by/4.0/.

\section{References}

1. Dittrich R, Kliesch S, Schüring A, Balcerek M, BastonBüst DM, Beck R, et al. Fertility preservation for patients with malignant disease. Guideline of the DGGG, DGU and DGRM (S2k-level, AWMF registry no. 015/082, November 2017) - recommendations and statements for girls and women. Geburtshilfe Frauenheilkd. 2018;78:567-84.

2. Dolmans M-M, Manavella DD. Recent advances in fertility preservation. J Obstet Gynaecol Res. 2019;45:266-79.

3. Cakmak H, Katz A, Cedars MI, Rosen MP. Effective method for emergency fertility preservation: random-start controlled ovarian stimulation. Fertil Steril. 2013;100:1673-80.

4. von Wolff M, Capp E, Jauckus J, Strowitzki T, Germeyer A. FertiPROTEKT study group. Timing of ovarian stimulation in patients prior to gonadotoxic therapy: an analysis of 684 stimulations. Eur J Obstet Gynecol Reprod Biol. 2016;199:146-9.

5. Azim AA, Costantini-Ferrando M, Oktay K. Safety of fertility preservation by ovarian stimulation with letrozole and gonadotropins in patients with breast cancer: a prospective controlled study. JClin Oncol. 2008;26:2630-5.

6. Lawrenz B, Jauckus J, Kupka M, Strowitzki T, von WolffM. Efficacy and safety of ovarian stimulation before chemotherapyin 205 cases. Fertil Steril. 2010;94:2871-3.

7. Cao Y-X, Chian R-C. Fertility preservation with immature and in vitro matured oocytes. Semin Reprod Med. 2009;27:456-64.

8. Donnez J, Dolmans M-M. Fertility Preservation in Women. NEngl J Med. 2017;377:1657-65.

9. Dolmans M-M, Masciangelo R. Risk of transplanting malignant cells in cryopreserved ovarian tissue. Minerva Ginecol. 2018;70:436-43.

10. DonnezJ, Dolmans M-M, PellicerA, Diaz-GarciaC, Sanchez Serrano M, Schmidt KT, et al. Restoration of ovarian activity and pregnancy after transplantation of cryopreserved ovarian tissue: a review of 60 cases of reimplantation. Fertil Steril. 2013;99:1503-13.

11. Chian R-C, Uzelac PS, Nargund G. In vitro maturation of human immature oocytes for fertility preservation. Fertil Steril. 2013;99:1173-81.

12. Winkler-Crepaz K, Böttcher B, Toth B, Wildt L, HoferTollinger S. What is new in 2017? Update on fertility preservation in cancer patients. Minerva Endocrinol. 2017;42:331-9.

13. Wallace WHB, Thomson AB, Saran F, Kelsey TW. Predicting age of ovarian failure after radiation to a field that includes the ovaries. Int J Radiat Oncol Biol Phys. 2005;62:738-44. 
14. Hoekman EJ, BroedersEABJ, Louwe LA, NoutRA, Jansen FW, de Kroon CD. Ovarian function after ovarian transposition andadditional pelvic radiotherapy: asystematic review. Eur JSurg Oncol. 2019;45:1328-40.

15. IrtanS, OrbachD, HelfreS, SarnackiS.Ovarian transposition in prepubescent and adolescent girls with cancer. Lancet Oncol. 2013;14:e601-e8.

16. Practice Committee of the American Society for Reproductive Medicine. Fertility preservation in patients undergoing gonadotoxic therapy or gonadectomy: a committee opinion. Fertil Steril. 2019;112:1022-33.

17. Hickman LC, Llarena NC, Valentine LN, Liu X, Falcone T. Preservation of gonadal function in women undergoing chemotherapy: a systematic review and meta-analysis of the potential role for gonadotropin-releasing hormone agonists. JAssist Reprod Genet. 2018;35:571-81.

18. Demeestere I, Brice P, Peccatori FA, Kentos A, Dupuis J, Zachee P, et al. No evidence for the benefit of gonadotropinreleasing hormone agonist in preserving ovarian function and fertility in lymphoma survivors treated with chemotherapy: final long-term report of a prospective randomized trial. J Clin Oncol. 2016;34:2568-74.

19. MooreHCF, UngerJM, Phillips K-A, BoyleF, HitreE, Porter D, et al. Goserelin for ovarian protection during breast-cancer adjuvant chemotherapy. NEngl J Med. 2015;372:923-32.

20. Lambertini M, Boni L, Michelotti A, Gamucci T, Scotto T, Gori S, et al. Ovarian suppression with triptorelin during adjuvant breast cancer chemotherapy and long-term ovarian function, pregnancies, and disease-free survival: a randomized clinical trial. JAMA. 2015;314:2632-40.
21. Chen H, Xiao L, Li J, Cui L, Huang W. Adjuvant gonadotropin-releasing hormone analogues for the prevention of chemotherapy-induced premature ovarian failure in premenopausal women. Cochrane Database Syst Rev. 2019; https://doi.org/10.1002/14651858.CD008018.pub3.

22. Loren AW, Mangu PB, Beck LN, Brennan L, Magdalinski AJ, Partridge AH, et al. Fertility preservation for patients with cancer: American Society of Clinical Oncologyclinical practice guideline update. J Clin Oncol. 2013;31(19):2500-10. https://doi.org/10.1200/JCO.2013.49.2678.

23. Tschudin S, Bitzer J. Psychological aspects of fertility preservation in men and women affected by cancer and other life-threatening diseases. Hum Reprod Update. 2009;15:587-97.

24. Böttcher B, Paul N. Personale Autonomie: Diskussion eines zentralen ethischen Konzepts am Beispiel von fertilitätsprotektiven Maßnahmen bei Krebspatientinnen. Ethik Med. 2013;25:47-54.

Publisher's Note Springer Nature remains neutral with regard to jurisdictional claims in published maps and institutional affiliations.

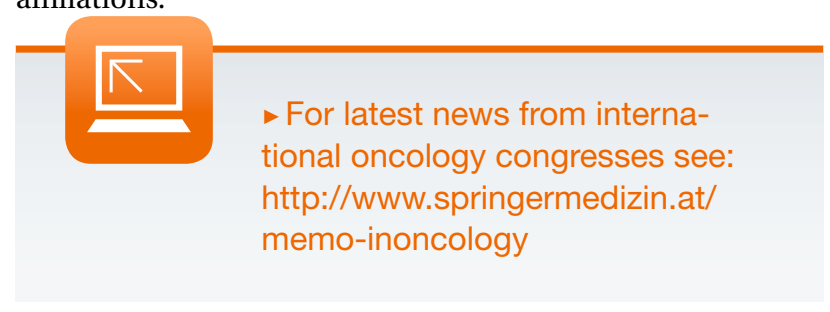

